DRAFT VERSION APRIL 22, 2018

Preprint typeset using LTEX style emulateapj v. 5/2/11

\title{
DETECTIONS OF PLANETS IN BINARIES THROUGH THE CHANNEL OF CHANG-REFSDAL GRAVITATIONAL LENSING EVENTS
}

\author{
Cheongho HAN ${ }^{1}$, In-Gu Shin ${ }^{2}$, Youn KIL JUnG ${ }^{2}$ \\ ${ }^{1}$ Department of Physics, Chungbuk National University, Cheongju 361-763, Republic of Korea and \\ ${ }^{2}$ Harvard-Smithsonian Center for Astrophysics, 60 Garden St., Cambridge, MA, 02138, USA \\ Draft version April 22, 2018
}

\begin{abstract}
Chang-Refsdal (C-R) lensing, which refers to the gravitational lensing of a point mass perturbed by a constant external shear, provides a good approximation in describing lensing behaviors of either a very wide or a very close binary lens. C-R lensing events, which are identified by short-term anomalies near the peak of a highmagnification lensing light curves, are routinely detected from lensing surveys, but not much attention is paid to them. In this paper, we point out that C-R lensing events provide an important channel to detect planets in binaries, both in close and wide binary systems. Detecting planets through the $\mathrm{C}-\mathrm{R}$ lensing event channel is possible because the planet-induced perturbation occurs in the same region of the C-R lensing-induced anomaly and thus the existence of the planet can be identified by the additional deviation in the central perturbation. By presenting the analysis of the actually observed C-R lensing event OGLE-2015-BLG-1319, we demonstrate that dense and high-precision coverage of a C-R lensing-induced perturbation can provide a strong constraint on the existence of a planet in the wide range of the planet parameters. The sample of an increased number of microlensing planets in binary systems will provide important observational constraints in giving shape to the details of the planet formation scenario which has been restricted to the case of single stars.
\end{abstract}

Subject headings: gravitational lensing: micro - planetary systems

\section{INTRODUCTION}

Majority of stars reside in binary systems $\mathrm{Abt} 1983$; Raghavan et al. 2010). The mechanism of planet formation around binary systems would be different from that around single stars not only because the environment of the protoplanetary disk would be affected by the binary companion but also because the binary companion would affect the long-term stability of the planet orbit. Therefore, one of the most generic environments to be considered in the study of planet formation should be that of a binary. However, the major planet-formation scenarios that have been developed over the past decades, e.g., core-accretion theory (Safronov 1969; Goldreich \& Ward 1973; Hayashi et al. 1985; Pollack et al. 1996) and disk instability theory (Kuiper 1951; Cameron 1978; Boss 2012), were mostly focused on the case of single stars.

Although a considerable work has been done about the effect of binary companions on the planet formation (e.g., Thebault \& Haghighipour 2015) and the long-term orbital stability (e.g., Szebehely 1980), this work was restricted to mostly theoretical studies and thus many details about the planet formation scenario remain uncertain. These details can be refined by the constraints provided by the sample of actually detected planetary systems. Unfortunately, there exists only a total of 19 known planets in 17 binary systems and most of these planets exist under a similar environment, i.e. circumbinary planets orbiting very close binaries. To give details about the planet formation in binary systems, therefore, it is important to detect more of such planets residing under various environments.

When a gravitational microlensing event is caused by a very wide binary object, the lensing behavior in the region around each lens component is approximated by ChangRefsdal (C-R) lensing, which refers to the gravitational lensing of a point mass perturbed by a constant external shear $\gamma$ (Chang \& Refsdal 1979, 1984). In the low shear regime $(\gamma<1)$, C-R lensing induces a small astroid-shape caustic around the lens. The lensing behavior of a very close binary, on the other hand, can be approximated by a point-mass plus quadrupole lensing. In this case, an astroidal caustic similar to the $\mathrm{C}-\mathrm{R}$ lensing caustic is produced around the center of mass of the binary. For this reason, the event caused by a very wide or a very close binary lens is often referred to as the C-R lensing event. Due to the existence of the caustic in the central region, $\mathrm{C}-\mathrm{R}$ lensing events are identified by a short-term anomaly that appears near the peak of a very highmagnification event. Although C-R lensing events are routinely detected from microlensing surveys, not much attention is paid to them because they are thought to be simply one type of numerous binary-lens events and thus of little scientific importance.

In this work, we point out that $\mathrm{C}$-R lensing events provide an important channel to detect planets in binaries, both in close and wide binary systems. In order to demonstrate that dense and high-precision coverage of a C-R lensing-induced perturbation can provide a strong constraint on the existence of a planet in the wide range of the planet parameters, we present analysis of the actually observed C-R lensing event OGLE-2015-BLG-1319.

The paper is organized as follows. In section 2, we briefly describe the lensing properties in the cases where the lens is composed of a single, binary, and triple masses. We also describe the $\mathrm{C}-\mathrm{R}$ lensing behavior. In section 3 , we estimate the detection efficiency of planets in binaries by conducting analysis of the lensing event OGLE-2015-BLG-1319. We summarize the results and conclude in section 4.

2. CHANG-REFSDAL LENSING CHANNEL 
When a point-mass lensing event occurs the lensing behavior is described by the lens equation

$$
\zeta=z-\frac{1}{\bar{z}}
$$

where $\zeta=\xi+i \eta$ and $z=x+i y$ denote the complex notations of the source and image positions, respectively, and $\bar{z}$ represents the complex conjugate of $z$. Here all lengths are normalized to the angular Einstein ring radius $\theta_{\mathrm{E}}$ and the lens is positioned at the origin. Solving the lens equation yields two solutions of image positions: one outside and the other inside the Einstein ring. The magnification $A_{j}$ of each image $j$ is given by

$$
A_{j}=\frac{1}{\operatorname{det} J_{j}} ; \quad \operatorname{det} J_{j}=\left|1-\frac{\partial \zeta}{\partial \bar{z}} \frac{\partial \bar{\zeta}}{\partial z}\right|_{z=z_{j}},
$$

where $J_{j}$ is the Jacobian of the lens equation evaluated at the image positions $z_{j}$ and $\operatorname{det} J_{j}$ is the determinant of the Jacobian. Since the individual microlensing images cannot be resolved, the observed lensing magnification is the sum of the magnifications of the individual images, i.e. $A=\sum_{i} A_{i}$. For a point mass, the magnification is represented analytically by

$$
A=\frac{|\zeta|^{2}+2}{|\zeta| \sqrt{|\zeta|^{2}+4}}
$$

where $|\zeta|=\left(t-t_{0}\right) / t_{\mathrm{E}}+i u_{0}$ is the lens-source separation with the length normalized to $\theta_{\mathrm{E}}, t_{\mathrm{E}}$ is the Einstein time scale, $t_{0}$ is the time of the closest lens-source approach, and $u_{0}$ is the lens-source separation at $t_{0}$. For a rectilinear relative lenssource motion, the lensing light curve is characterized by a smooth and symmetric shape (Paczyński 1986).

When an event is produced by a lens composed multiple components, the lens equation is expressed as

$$
\zeta=z-\sum_{i=1}^{N} \frac{\epsilon_{i}}{\bar{z}-\bar{z}_{L, i}}
$$

where $z_{L, i}$ and $\epsilon_{i}=m_{i} / m_{\text {tot }}$, and $m_{i}$ represent the location, mass fraction, and mass of each lens component, respectively. The notation $N$ denotes the number of the lens components, and thus $N=2$ for a binary lens. Here all lengths ( $\zeta$ and $z$ 's) are in units of the angular Einstein radius corresponding to the total mass of the lens, $\theta_{\mathrm{E}}$. One of the most important properties of a binary lens that differentiate from those of a single lens is the formation of caustics, which represent the sets of source positions at which $\operatorname{det} J=0$ and thus the magnification of a point source becomes infinite. As a result, a binary-lensing light curve can exhibit strong deviations when a source approaches close to or passes over the caustic. Caustics of a binary lens form a single or multiple sets of closed curves and each curve composed of concave curves that meet at cusps. The topology of the binary-lens caustic is broadly classified into 3 categories (Erdl \& Schneider 1993; Daněk \& Heyrovský 2015). In the case of a binary where the binary separation is greater than $\left(\sqrt[3]{\epsilon_{1}}+\sqrt[3]{\epsilon_{2}}\right)^{3 / 2}$ (wide binary), there exist two sets of 4-cusp caustics that are located close to the individual lens components. When the binary separation is smaller than $\left(\sqrt[3]{\epsilon_{1}}+\sqrt[3]{\epsilon_{2}}\right)^{-3 / 4}$ (close binary), the caustic is composed of 3 pieces, where the central caustic with 4 cusps is formed around the center of mass of the binary lens, and the other 2 triangular caustics are located away from the center of mass. In the intermediate separation region, there exists a single big caustic with 6 cusps. For the visual presentation of the
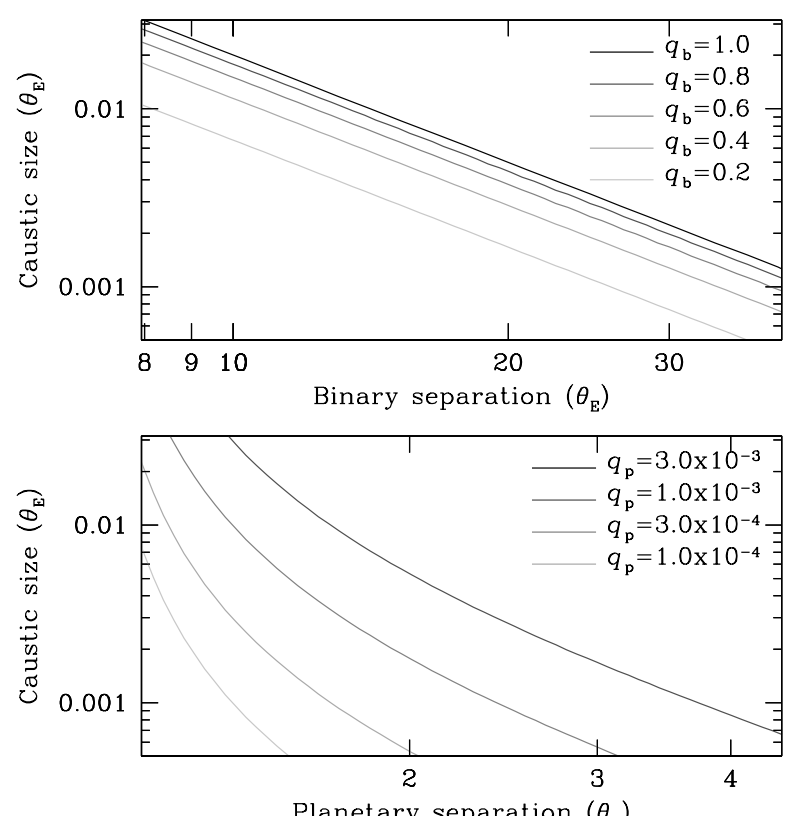

FIG. 1.- Sizes of the C-R lensing caustic (upper panel) and the planetinduced central caustic as a function of the separation between the lens components. Curves with different grey tone show the variation of the caustic size depending on the mass ratio between binary lens components $q_{b}$ (upper panel) and the mass ratio between the planet and the host $q_{p}$ (lower panel). Both the caustic size and the binary separation are normalized to the angular Einstein radius corresponding to the total lens mass.

binary caustic topology, see Figure 3 of Dominik (1999). In the extreme case where the binary separation is much greater or less than $\theta_{\mathrm{E}}$, the 4-cusp central caustic has an astroid shape which is symmetric with respect to the binary axis and the line vertical to the binary axis.

For the exact description of the lensing behavior produced by a lens system of a planet orbiting a binary object, one needs the triple lens equation, i.e. Eq. (4) with $N=3$. With the addition of a third lens component, the complexity of the lensing behavior greatly increases and caustics can exhibit self-intersection and nesting (Rhie 1997; Gaudi et al. 1998; Daněk \& Heyrovský 2015).

A planetary lens corresponds to the extreme case of a binary lens where the mass of one of the lens components is much smaller than the other, i.e. $\epsilon_{1} \sim 1$ and $\epsilon_{2} \ll 1$. In this case, the lens equation is approximated as

$$
\zeta \sim z-\frac{1}{\bar{z}}-\frac{q_{p}}{\bar{z}-\bar{z}_{p}},
$$

where $q_{p}=m_{2} / m_{1}$ is the mass ratio between the planet and the host and $z_{p}$ represents the position of the planet with respect to the host. We note that the lengths of $\zeta$ and $z$ 's in Eq. (5) are normalized to the angular Einstein radius corresponding to the mass of the primary lens, i.e. $\theta_{\mathrm{E}, 1}=\theta_{\mathrm{E}} /\left(1+q_{p}\right)^{1 / 2} \sim$ $\left(1-q_{p} / 2\right) \theta_{\mathrm{E}}$. For a planetary lens, however, the mass of the primary dominates, i.e. $q_{p} \ll 1$, and thus $\theta_{\mathrm{E}, 1} \sim \theta_{\mathrm{E}}$. The planet induces two types of caustics, where one is located close to the host (central caustic) and the other is away from the host. The central caustic has an arrowhead shape and its size is related to the star-planet separation $s_{p}=\left|z_{p}\right|$ and the mass ratio by (Chung et al. 2005)

$$
\Delta x_{\mathrm{p}}=\frac{4 q_{p}}{\left(s_{p}-s_{p}^{-1}\right)^{2}} .
$$


Due to the location of the central caustic close to the host, perturbations induced by the central caustic of a planet always appear near the peak of the lensing light curve produced by the host of the planet (Griest \& Safizadeh 1998).

The lens equation of C-R lensing is expressed as

$$
\zeta=z-\frac{1}{\bar{z}}+\gamma \bar{z}
$$

which describes the lensing behavior around the primary lens with an external shear $\gamma$. Here lengths are given in units of the Einstein radius corresponding to the primary mass. The shear induces a caustic around the lens. The shape of the caustic is similar to the central caustic of a very wide binary lens and thus the C-R lensing provides a good approximation in describing the binary lensing behavior in the region around the caustic. For a very close binary lens, the lensing behavior around the center of mass is described by quadrupole lensing (Dominik 1999), which also induces an astroidal caustic similar to the C-R lensing caustic. To the first order approximation, the caustic size in units of the angular Einstein radius of the binary-lens mass, $\theta_{\mathrm{E}}$, is related to the separation and the mass ratio between the binary-lens components by

$$
\Delta x_{\mathrm{C}-\mathrm{R}}=\frac{4 \gamma}{\sqrt{1-\gamma}} ; \quad \gamma=\frac{q_{b}}{s_{b}^{2}\left(1+q_{b}\right)},
$$

for a wide binary and

$$
\Delta x_{\text {quad }}=4 Q\left(1+\frac{9}{2} Q\right) ; \quad Q=\frac{s_{b}^{2} q_{b}}{\left(1+q_{b}\right)^{2}}
$$

for a close binary.

A C-R lensing event can provide a channel to detect planets in binary systems. This is because both of the C-R lensing caustic and the central caustic induced by the planet occur in the same region around the primary lens and the size of the planet-induced central caustic can be comparable to the size of the C-R lensing caustic (Lee et al. 2008). In Figure 1, we present the sizes of the C-R lensing caustic and the planet-induced central caustic as a function of the primarycompanion separation. We note that a pair of caustics with separations $s$ and $s^{-1}$ have the same size to a linear order and thus we present distributions for only wide binaries. The plot shows that the central caustic induced by planets located in the "lensing zone' $\square$ is of considerable size compared to the size of the C-R lensing caustic. This suggests that the C-R lensing anomaly can be additionally affected by the planetary perturbation, enabling one to identify the presence of a planet in the binary system. In Figure 2, we present the variation of the C-R lensing caustic and the lensing light curve affected by the presence of planets with various separations and mass ratios between the planet and the primary of the binary. See Luhn et al. (2016) for more details about the caustic variation.

We note that the C-R lensing channel enables detections of planets not only in close binaries but also in wide binaries. To be dynamically stable, a planet should be either in a circumbinary (or P-type) orbit, where the planet orbits the barycenter of the two stars of a close binary, or in a circumprimary (S-type) orbit, where the planet orbits just one star

1 The lensing zone represents the range of the planet-host separations where the probability of detecting the planet is high Gould \& Loeb 1992; Griest \& Safizadeh 1998). The range is approximately $1 / 2 \lesssim s \lesssim 2$, although the range varies depending on how the lensing zone is defined. of a wide binary system. This condition of the planet existence in the binary system matches the lens system configuration of the proposed C-R lensing channel of planet detections. Planets in binary systems can be detected by various methods such as transit (e.g., Doyle et al. 2011), eclipsing binary timing (e.g., Oian et al. 2010), and radial-velocity methods (e.g., Correia et al. 2005). Due to the intrinsic nature of the methods, however, it is difficult to detect planets in circumprimary orbits and thus all planets in binaries detected by these methods, 19 in total, reside in circumbinary orbits. On the other hand, C-R lensing events can be produced by both close and wide binary systems and thus the proposed C-R lensing channel provides a unique channel to detect planets both in circumbinary and circumprimary orbits 2

\section{PLANET DETECTION EFFICIENCY}

In this section, we demonstrate the high efficiency of the proposed C-R lensing channel in detecting planets of binary systems. Estimating the efficiency requires to consider various details of observational conditions such as photometric precision and cadence. In order to reflect realistic observational conditions, we estimate the detection efficiency for an example C-R lensing event that was actually observed by lensing experiments.

The event used for our efficiency estimation is OGLE2015-BLG-1319. The event was analyzed in detail by Shvartzvald et al. (2016) and turned out to be an exemplary $\mathrm{C}-\mathrm{R}$ lensing event caused either by a close or a wide binary lens. Figure 3 shows the light curve of the event reproduced based on the same data sets as those used in the previous analysis. The model light curve superposed on the data points is obtained from binary-lensing modeling based on one of the 8 degenerate solutions ("++ wide" solution) presented in Shvartzvald et al. (2016). The inset in the lower panel shows the source trajectory with respect to the caustic, which has a very characteristic shape of a C-R lensing caustic. The anomaly caused by the C-R lensing caustic in the peak of the light curve was densely and precisely observed. This was possible because the event was predicted to be a high-magnification event before it reached the peak based on the light curve obtained from the survey observation conducted by the Optical Gravitational Lens Experiment (OGLE: Udalski 2003) and the Microlensing Observations in Astrophysics (MOA: Bond et al. 2001; Sumi et al. 2003), enabling intensive observations of the peak by follow-up observation groups including the Microlensing Follow-Up Network $(\mu$ FUN: Gould et al. 2006) and the RoboNet (Tsapras et al. 2009) groups. We note that the event was also observed in space using two space telescopes Spitzer and Swift. This enabled the determinations of the lens mass and the distance, but we do not use the space-based data because these data are irrelevant to our scientific purpose. The values of the binary separation and the mass ratio presented in Shvartzvald et al. (2016) are $\left(s_{b}, q_{b}\right) \sim(0.08,0.07)$ for the close binary solution and and $\left(s_{b}, q_{b}\right) \sim(14,0.09)$ for the wide solution. For other lensing parameters, see Table 1 of Shvartzvald et al. (2016).

To show the constraint on the existence of a planet, we construct an "exclusion diagram", which shows the proba-

\footnotetext{
${ }^{2}$ We note that there exist two known planets in wide binary systems. These planets, OGLE-2013-BLG-0341LBb (Gould et al. 2014) and OGLE-2008BLG-092LAb (Poleski et al. 2014), were detected by using the microlensing method. However, they were detected not through the C-R lensing channel but through a repeating channel where the perturbations induced by the planet and the binary companion were separately detected.
} 

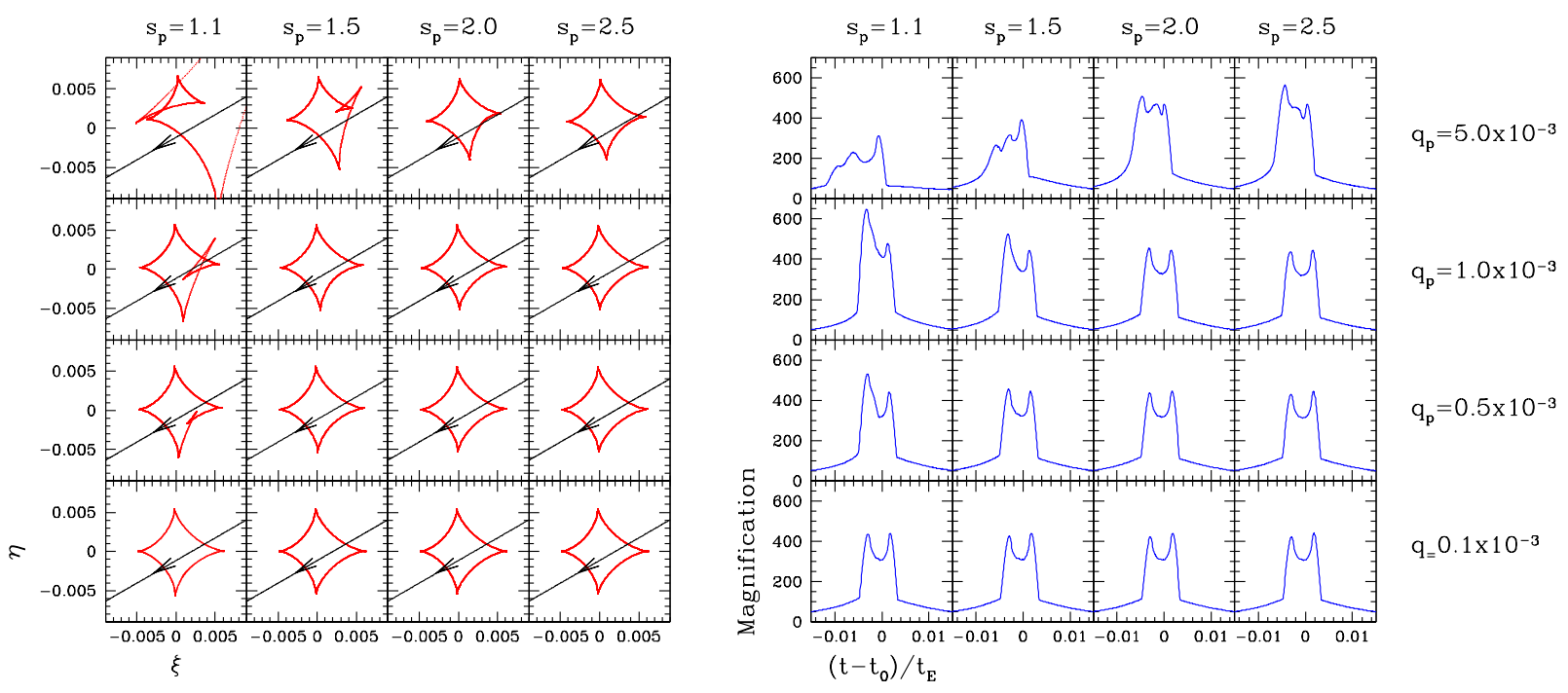

FIG. 2.- Variation of the C-R lensing caustic (left panel) and the light curve (right panel) by the presence of planets with various separations $s_{p}$ and the mass ratios $q_{p}$. The C-R lensing caustic is produced by a binary companion with $s_{b}=10$ and $q_{b}=0.5$. The separations $s_{p}$ and $s_{b}$ are normalized to the angular Einstein radius corresponding to the total lens mass, $\theta_{\mathrm{E}}$. The light curve in each subpanel results from the source trajectory (line with an arrow) marked in the corresponding subpanel showing the caustic. The notations $(\xi, \eta)$ represent the coordinates on the source plane where $\xi$ is aligned with the binary-lens axis and lengths are normalized to $\theta_{\mathrm{E}}$. The planet is located at $\left(x_{p}, y_{p}\right)=\left(x_{1}+s_{p} \cos \phi, y_{1}+s_{p} \sin \phi\right)$, where $\left(x_{1}, y_{1}\right)$ is the position of the primary lens (heavier binary component) and $\phi=60^{\circ}$ is orientation angle of the planet with respect to the binary axis.
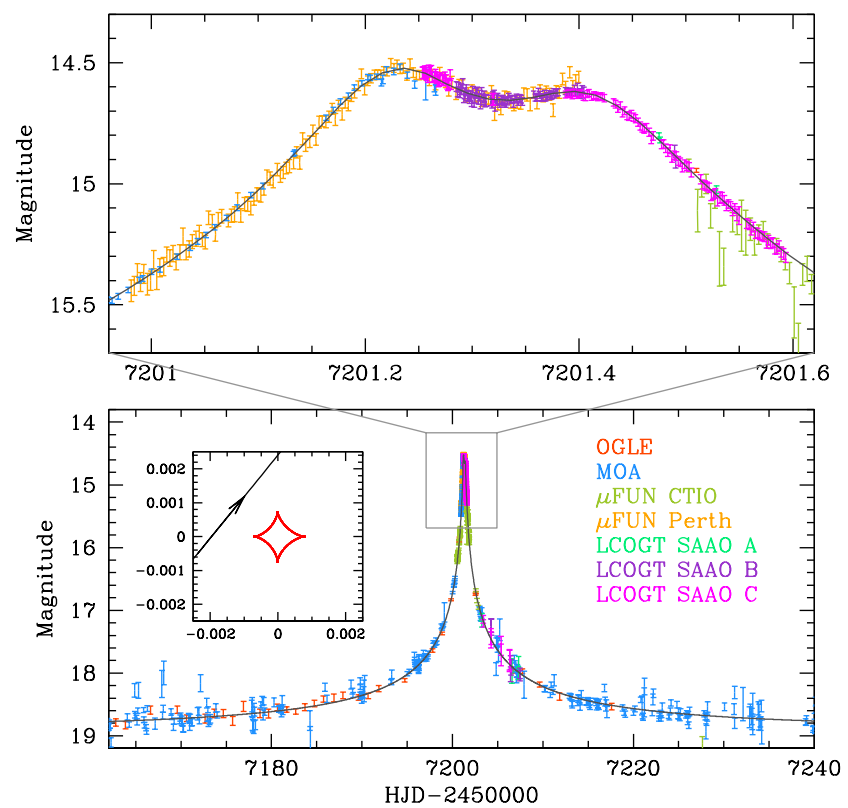

FIG. 3.- Light curve of the microlensing event OGLE-2015-BLG-1319. The lower and upper panels show the whole view of the light curve and the zoom around the peak. The inset in the lower panel shows the source trajectory with respect to the caustic. The label in the legend denote the lensing experiments and the telescopes used for observations.

bility of excluding the existence of a planet as a function of the planet separation and the mass ratio (Albrowet al. 2000; Gaudi \& Sackett 2000; Kubas et al. 2008; Gould et al. 2010; Cassan et al. 2012). We construct the exclusion diagram following the procedure of Shin et al. (2015). In this procedure, we first introduce a planet with the parameters $\left(s_{p}, q_{p}, \psi\right)$ to the binary lens with the parameters $\left(s_{b}, q_{b}\right)$. Here $\left(s_{b}, q_{b}\right)$ represent the separation and the mass ratio between the binary components, while $\left(s_{p}, q_{p}\right)$ are the separation and the mass ratio between the primary of the binary and the planet. The an- gle $\psi$ denotes the orientation angle of the planet with respect to the binary axis connecting the binary lens components. We use $\left(s_{b}, q_{b}\right)$ that are determined from the binary-lensing modeling. For a given set of $\left(s_{p}, q_{p}, \psi\right)$, we then search for other parameters that yield the best fit to the observed light curve and compute $\chi^{2}$ of the fit. We repeat this process for many different orientation angles. Then, the probability of excluding a planet for a given $\left(s_{p}, q_{p}\right)$ is estimated as the fraction of the angles $\psi$ that result in fits with $\Delta \chi^{2}>\Delta \chi_{\text {th }}^{2}$, where $\Delta \chi^{2}$ is the difference between the triple-lens (i.e. binary + planet) and the binary-lens models. As a criteria for the planet detection, we adopt a threshold value of $\Delta \chi_{\mathrm{th}}^{2}=500$, which is a generally agreed value for planets detected through the highmagnification channel (Gould et al. 2010). The probability of excluding a planet corresponds to the probability of detecting the planet, i.e. planet detection efficiency.

Figure 4 shows the constructed exclusion diagram which shows the planet detection efficiency as a function of the normalized separation $s_{p}$ and the mass ratio $q_{p}$ of the planet. Since the microlens parallax of the event OGLE-2015-BLG1319 was determined using the combined data taken from the ground and space, the physical sizes of the primary-planet separation and the mass of the planet were determined. By adopting the lens mass of $M_{\mathrm{tot}}=0.62 M_{\odot}$, the angular Einstein radius of $\theta_{\mathrm{E}}=0.63$ mas, and the distance to the lens of $D_{\mathrm{L}}=4.93 \mathrm{kpc}$, that were determined by Shvartzvald et al. (2016), i.e. $(+,+)$ wide model, we convert $s_{p}$ and $q_{p}$ into the physical sizes of the primary-planet separation in au, i.e. $a_{\perp}=s_{p} D_{\mathrm{L}} \theta_{\mathrm{E}}$, and the mass of the planet in Jupiter masses $\left(M_{J}\right)$, i.e. $M_{p}=q M$, and they are presented in the upper $x$ axis and the right of the $y$ axis, respectively.

In Table 1, we present the ranges of planet detection for 3 different planets with masses corresponding to those of the Jupiter, Saturn $\left(\sim 0.3 M_{J}\right)$, and Neptune $\left(\sim 0.055 M_{J}\right)$ of the Solar system. It is found that the detection efficiency is greater than $90 \%$ for the Jupiter- and Saturn-mass planets located in the ranges of $1.0-10$ au and $2.0-5$ au from the host, re- 


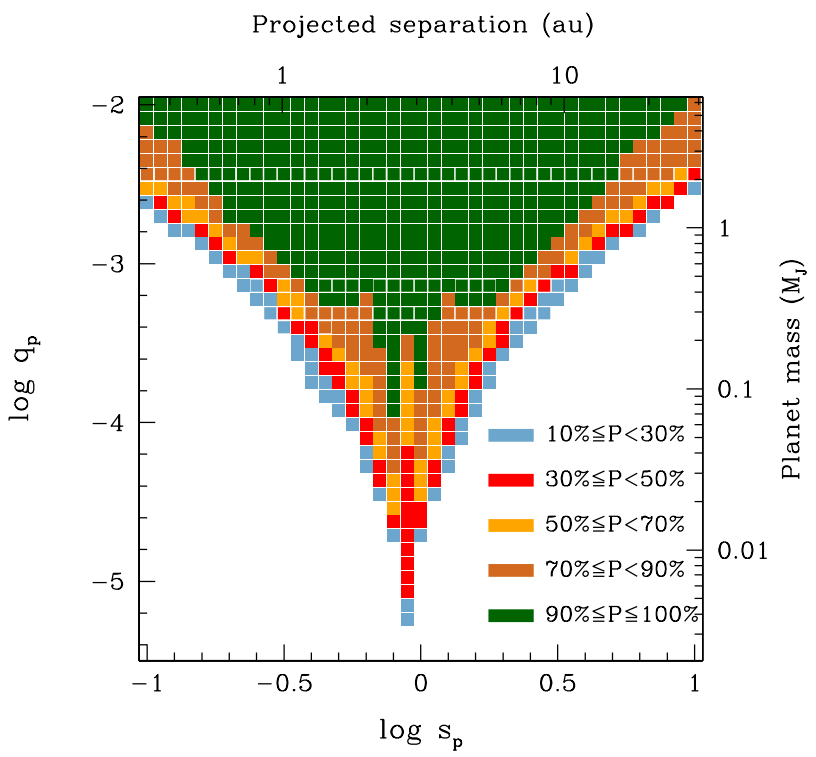

FIG. 4.- Efficiency of detecting planets in the binary lens system responsible for the lensing event OGLE-2015-BLG-1319 as a function of the normalized separation $s_{p}$ and the mass ratio $q_{p}$ between the planet and the primary of the binary lens. The values marked in the upper $x$ axis and the right of $y$ axis represent the physical primary-planet separation in au and the mass of the planet in Jupiter masses, respectively. The color coding represents the regions of different efficiencies that are marked in the legend. The planetary separation is expressed in units of the angular Einstein radius corresponding to the binary lens mass.

TABLE 1

RANGE OF PLANET DETECTION

\begin{tabular}{cccc}
\hline \hline $\begin{array}{c}\text { Planet } \\
\text { type }\end{array}$ & $>90 \%$ & $\begin{array}{c}\text { Efficiency } \\
>50 \%\end{array}$ & $>10 \%$ \\
\hline Jupiter & $1.0-10 \mathrm{au}$ & $0.5-11 \mathrm{au}$ & $0.4-18 \mathrm{au}$ \\
Saturn & $2.0-5 \mathrm{au}$ & $1.0-7 \mathrm{au}$ & $0.9-9 \mathrm{au}$ \\
Neptune & - & $2.0-3.5 \mathrm{au}$ & $1.8-4.0 \mathrm{au}$ \\
\hline
\end{tabular}

spectively. The ranges with $>10 \%$ probability are $0.4-18$ au, $0.9-9$ au, and 1.8 - 4 au for the Jupiter-, Saturn-, and Neptune-mass planets, respecively. These ranges encompass wide regions around snow lines where giants are believed to form.

Despite the high efficiency of C-R lensing events in detecting planets of binary systems, there exists no report of planet detection yet. The reasons for this can be (1) the rarity of C$\mathrm{R}$ lensing events, (2) the unoptimized observational strategy for planet detections, and (3) the rarity of planets in the region of sensitivity. Considering the existence of the already known microlensing planets detected through the repeatingevent channel and those detected by other methods combined with the wide region of planet sensitivity of the C-R lensing channel, the reason (3) is unlikely to be the main reason of nondetection. To check the possibilities (1) and (2), we investigate the lensing events reported by the OGLE and MOA surveys in 2015 season. From the systematic analyses of all highmagnification events with anomalies near the peaks based on the online data of the surveys, we find $12 \mathrm{C}-\mathrm{R}$ lensing events including OGLE-2015-BLG-1319, MOA-2015-BLG040/OGLE-2015-BLG-0318, OGLE-2015-BLG-0697/MOA2015-BLG-148, OGLE-2015-BLG-0797, OGLE-2015-BLG0812, OGLE-2015-BLG-0189 MOA-2015-BLG-085/OGLE2015-BLG-0472, OGLE-2015-BLG-0313/MOA-2015-BLG047, OGLE-2015-BLG-0033/MOA-2015-BLG-017, MOA2015-BLG-047, OGLE-2015-BLG-0919, and OGLE-2015BLG-0863. This indicates that the rarity of C-R lensing events is not the reason for the nondetection, either. However, we find that the coverage of the peak regions for all of the C-R lensing events except OGLE-2015-BLG-1319 was not dense enough to constrain the existence of a planet. Therefore, it is likely that the main reason for the nondetection of planet is due to the unoptimized observational strategy. In other words, this suggests that planets can be detected through the proposed channel in abundance with an aggressive strategy to densely cover the peak regions of high-magnification events, e.g., vigilant monitoring of high-magnification events and timely alerts of anomalies followed by prompt and intensive coverage of the anomalies by follow-up observations

\section{SUMMARY AND CONCLUSION}

We pointed out that C-R lensing events could provide one with an important channel to detect planets in binary systems. We also pointed out that while other methods could detect planets only in circumbinary orbits the proposed C-R lensing channel could provide a unique channel to detect planets both in circumbinary and circumprimary orbits. We demonstrated the high sensitivity of the C-R lensing channel to planets in a wide range of planet parameter space by presenting the exclusion diagram for an actually observed C-R lensing event. We mentioned that an increased number of microlensing planets in binary systems could be detected with an aggressive strategy to densely cover the peak regions of high-magnification events. The sample of an increased number of microlensing planets in binaries will make it possible to provide important observational constraints that can give shape to the details of the formation scenario which has been restricted to the case of single stars.

Work by C. Han was supported by the Creative Research Initiative Program (2009-0081561) of National Research Foundation of Korea. We acknowledge the high-speed internet service (KREONET) provided by Korea Institute of Science and Technology Information (KISTI).

\section{REFERENCES}

Abt, H. A. 1983, ARA\&A, 21, 343

Albrow, M. D., Beaulieu, J.-P., Caldwell, J. A. R., et al. 2000, ApJ, 535, 176

Bond, I. A., Abe, F., Dodd, R. J., et al. 2001, MNRAS, 327, 86

Boss, A. P. 2012, MNRAS, 419, 1930

Cameron, A. G. W. 1978, The Moon and the Planets, 18, 5

Cassan, A., Kubas, D., Beaulieu, J.-P., et al. 2012, Nature, 481, 167

Chang, K., \& Refsdal, S. 1979, Nature, 282, 561

Chang, K., \& Refsdal, S. 1984, Nature, 132, 168

Chung, S.-J., Han, C., Park, B.-G., et al. 2005, ApJ, 630, 535
Correia, A. C. M., Udry, S., Mayor, M., Laskar, J., Naef, D., Pepe, F.,

Queloz, D., \& Santos, N. 2005, A\&A, 440, 751

Daněk, K., \& Heyrovský, D. 2015, ApJ, 806, 99

Dominik, M. 1999, A\&A, 349, 108

Doyle, L. R., Carter, J. A., Fabrycky, D. C., et al. 2011, Science, 333, 1602

Erdl H., \& Schneider P. 1993, A\&A, 268, 453

Gaudi, B. S., Naber, R. M., \& Sackett, P. D. 1998, ApJ, 502, L33

Gaudi, B. S., \& Sackett, P. D. 2000, ApJ, 528, 5

Goldreich, P., \& Ward, W. 1973, ApJ, 183, 1051

Gould, A., \& Loeb, A. 1992, ApJ, 396, 104 
Gould, A., Dong, S., Gaudi, B. S., et al. 2010, ApJ, 720, 1073

Gould, A., Udalski, A., An, D., et al. 2006, ApJ, 644, 37

Gould, A., Udalski, A., Shin, I.-G., et al. 2014, Science, 345, 46

Griest, K., \& Safizadeh, N. 1998, ApJ, 500, 37

Hayashi, C., Nakazawa, K., \& Nakagawa Y. 1985, in Protostars and Planets II, eds. D. C. Black \& M. S. Matthew (Tucson: Univ. Arizona Press), 1100

Kim, S.-L., Lee, C.-U., Park, B.-G., et al. 2015, Journal of the Korean Astronomical Society, 49, 37

Kubas, D., Cassan, A., Dominik, M., et al. 2008, A\&A, 483, 317

Kuiper, G. P. 1951 Proc. Natl. Acad. Sci. U.S.A., 37, 1

Lee, D. W., Lee, C.-U., Park, B.-G., Chung, S.-J., Kim, Y.-S., Kim, H.-I., \& Han, C. 2008, ApJ, 672, 623

Luhn, J. K., Penny, M. T., \& Gaudi, B. S. 2016, ApJ, 827, 61

Paczyński, B. 1986, ApJ, 304, 1

Poleski, R., Skowron, J., Udalski, A., et al. 2014, ApJ, 795, 42

Pollack, J. B., Hubickyj, O., Bodenheimer, P., Lissauer, J. J., Podolak, M., \& Greenzweig, Y. 1996, Icarus, 124, 62
Qian, S.-B., Liu, L., Zhu, L.-Y., Dai, Z.-B., Lajús, E. F., \& Baume, G. L. 2010, MNRAS, 401, L34

Raghavan, D., McAlister, H. A., Henry, T. J., et al. 2010, ApJS, 190, 1

Rhie, S. H. 1997, ApJ, 484, 63

Safronov, V. 1969, Evolution of the Protoplanetary Cloud and Formation of the Earth and Planets (Moscow: Nauka)

Shin, I.-G., Han, C., Choi, J.-Y., Hwang, K.-H., Jung, Y.-K., \& Park, H. 2015, ApJ, 802,108

Shvartzvald, Y., Li, Z., Udalski, A., et al. 2016, ApJ, submitted

Sumi, T., Abe, F., Bond, I. A., et al. 2003, ApJ, 591, 204

Szebehely, V. 1980, Celest. Mech., 22, 7

Thebault, P., \& Haghighipour, N. 2015, Planetary Exploration and Science: Recent Results and Advances, eds. S. Jin, N. Haghighipour, W-H. Ip (Berlin: Springer), 309

Tsapras, Y., Street, R., Horne, K., et al. 2009, Astron. Nachr., 330, 4

Udalski, A. 2003, Acta Astron., 53, 291

Udalski, A., Szymański, M., Kalużny, J., Kubiak, M., \& Mateo, M. 1992, Acta Astron., 42, 253 